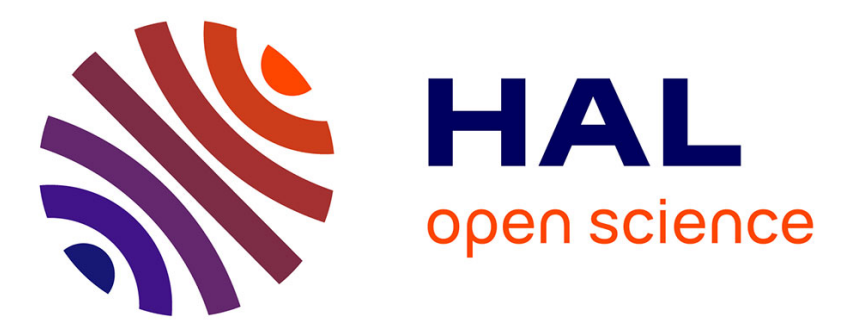

\title{
Stratégies de positionnement et trajectoires d'architectes Véronique Biau
}

\section{To cite this version:}

Véronique Biau. Stratégies de positionnement et trajectoires d'architectes. Sociétés contemporaines, 1998, 29, pp.7-25. 10.3406/socco.1998.1839 . halshs-01250692

\section{HAL Id: halshs-01250692 \\ https://shs.hal.science/halshs-01250692}

Submitted on 5 Jan 2016

HAL is a multi-disciplinary open access archive for the deposit and dissemination of scientific research documents, whether they are published or not. The documents may come from teaching and research institutions in France or abroad, or from public or private research centers.
L'archive ouverte pluridisciplinaire HAL, est destinée au dépôt et à la diffusion de documents scientifiques de niveau recherche, publiés ou non, émanant des établissements d'enseignement et de recherche français ou étrangers, des laboratoires publics ou privés. 


\title{
Stratégies de positionnement et trajectoires d'architectes
}

\section{Véronique Biau}

\begin{abstract}
Architects' occupational strategies and paths

The tension between "business» and "artistic creation» produces different occupa tional attitudes among architects. This paper focuses on the processes which attract architects in one direction or the other. The study of external communication prac tices reveals two opposite strategies, each targeted at a different type of clientele one consists in a fairly classical marketing strategy aiming at attracting and keeping a group of clients, whereas the second one involves an increasingly formalized careerpath aiming at winning public commissions. This latter path combines public archi tectural contests publications in professional media, and access to key positions. It might represent the archetype of what became a new form of canonic career, once the Academic system had fallen apart in 1968.
\end{abstract}

\section{Résumé}

En architecture, la dualité entre la logique de "faire des affaires» et celle de "réaliser des oeuvres» induit une diversité dans les positions professionnelles par rapport à la commande et à la reconnaissance symbolique. On s'intéresse ici aux dynamiques portant les architectes vers l'un ou l'autre de ces pôles. L'analyse des pratiques de communication externe des architectes fait apparaître deux familles de stratégies selon la clientèle recherchée: un marketing assez classique pour capter et fidéliser la clientèle privée d'une part, un parcours plus spécifique pour accéder à une commande publique dont l'attribution est de plus en plus codifiée d'autre part. Ce parcours articule étroitement les concours publics d'architecture, les publications dans la presse spécialisée et l'obtention de positions-clés dans l'enseignement et la critique. II pourrait constituer une forme renouvelée de carrière canonique de l'architecte, après la chute du système académique.

\section{Citer ce document / Cite this document :}

Biau Véronique. Stratégies de positionnement et trajectoires d'architectes. In: Sociétés contemporaines $\mathrm{N}^{\circ} 29$, 1998. pp. 7-25.

doi : $10.3406 /$ socco.1998.1839

http://www.persee.fr/doc/socco_1150-1944_1998_num_29_1_1839

Document généré le 21/10/2015

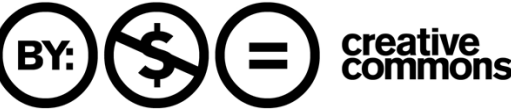




\title{
STRATÉGIES DE POSITIONNEMENT ET TRAJECTOIRES D'ARCHITECTES
}

\begin{abstract}
RÉSUMÉ : En architecture, la dualité entre la logique de "faire des affaires » et celle de "réaliser des cuvres » induit une diversité dans les positions professionnelles par rapport à la commande et à la reconnaissance symbolique. On s'intéresse ici aux dynamiques portant les architectes vers l'un ou l'autre de ces pôles. L'analyse des pratiques de communication externe des architectes fait apparaître deux familles de stratégies selon la clientèle recherchée : un marketing assez classique pour capter et fidéliser la clientèle privée d'une part, un parcours plus spécifique pour accéder à une commande publique dont l'attribution est de plus en plus codifiée d'autre part. Ce parcours articule étroitement les concours publics d'architecture, les publications dans la presse spécialisée et l'obtention de positions-clés dans l'enseignement et la critique. Il pourrait constituer une forme renouvelée de carrière canonique de l'architecte, après la chute du système académique.
\end{abstract}

Parce que l'architecture relève, comme on le verra, du paradoxe de «l'art sur commande », et qu'elle se trouve à un carrefour entre les logiques issues des milieux artistiques et intellectuels d'une part et celles des milieux technico-financiers de la construction d'autre part, diverses légitimités s'y croisent et s'y confrontent. Les architectes ont en effet à se situer par rapport à des processus bien connus dans les domaines artistiques comme l'innovation, la distinction par rapport aux confrères ou la consécration par la critique, mais aussi par rapport à des contraintes et des concurrences d'ordre strictement économique, dans la mesure où l'existence même de l'œuvre est dépendante du bon vouloir et des moyens matériels d'un commanditaire.

Par rapport à cette dualité constitutive de son objet, la recherche sociologique sur les architectes, dont l'histoire est encore relativement courte, a adopté des approches que l'on peut schématiquement ranger sous deux catégories complémentaires. Un premier pan de recherches s'attache à la division sociale et technique du travail et situe l'architecte au sein de la configuration de plus en plus étendue des acteurs à côté desquels il prend place dans ses tâches de conception et de production des espaces bâtis : ce sont alors les questions des rapports des architectes à leurs commanditaires d'une part, aux autres concepteurs d'autre part, aux entreprises de construction enfin qui sont posées, entraînant celles des missions, des contrats, des honoraires, des responsabilités, des compétences, etc. Un deuxième pan de travaux privilégie l'analyse de la dimension symbolique de l'activité architecturale, qu'il s'agisse de la perception et de l'évaluation de l'œuvre par son «public » et/ou ses

Sociétés Contemporaines (1998) $n^{\circ} 29(p .7-25)$ 
usagers ou bien des processus de reconnaissance voire de consécration des architectes les uns par rapport aux autres. Dans cette tentative d'élaborer un cadre global d'analyse de l'architecture comme champ de production culturelle, les premières balises ont été posées: un repérage a été effectué des principales instances structurantes, celles qui assurent la protection collective des intérêts des agents qui se trouvent dans ce champ, comme celles qui régulent les concurrences internes des individus pour l'accès aux différents pouvoirs et honneurs de la profession ${ }^{1}$. Par ailleurs, c'est la genèse et le sort de groupes quelque peu marginaux par rapport aux formes les plus légitimes d'exercice qui ont été étudiés : maîtres d'œuvre agréés, architectes de collectivités locales,... On pourrait citer encore des analyses de trajectoires individuelles ou des approches de processus révélateurs $\mathrm{du}$ fonctionnement du champ de l'architecture comme ceux de l'innovation.

Il ressort de ces travaux que ce groupe professionnel, bien qu'il soit ancien, profondément enraciné autour de traditions, de savoirs, de savoir-faire, d'institutions souvent centenaires voire multi-centenaires, et relativement restreint dans ses effectifs $^{2}$, ne peut pas être défini d'une manière univoque et homogène. Même si l'on ne prend en considération que la pratique cardinale de la profession, le projet et la réalisation d'édifices, (c'est-à-dire, en France où la maîtrise d'œuvre publique est inexistante, l'exercice en agence libérale ou en société), on se trouve chez les praticiens face à une multiplicité de représentations de leur profession, des enjeux et des savoir-faire qui la caractérisent. Cette multiplicité trouve d'ailleurs son reflet dans l'image de l'architecte auprès du grand public, qui voit en lui tout à la fois le grand créateur des édifices monumentaux contemporains et historiques, et le professionnel en lequel il place une confiance limitée pour intervenir sur sa propriété ou le cadre de sa vie quotidienne.

On dispose actuellement de quelques analyses permettant de situer les unes par rapport aux autres les principales positions occupées individuellement par les architectes dans cet espace, la principale dualité, pour la définir de façon sommaire, opposant des «architectes-artistes » à des «architectes-artisans » ou architectes «d'affaires ». Nous présenterons dans un premier temps une tentative de synthèse des caractéristiques de ces diverses positions, en les observant sur une période qui, même courte, voit considérablement varier les enjeux et les modalités de reconnaissance.

Mais cette vision globale de l'espace professionnel de l'architecture présente l'inconvénient de figer l'image des positions relatives, comme si les individus qui les occupent s'y étaient trouvés d'emblée, comme s'ils ne pouvaient pas être amenés au cours de leur carrière à passer de l'une à l'autre selon les flux et reflux de leur

1. Un certain nombre de travaux historiques ont aussi porté sur des instances comme l'Académie de France et le Prix de Rome, qui ont joué un rôle régulateur déterminant au cours des derniers siècles.

2. 26797 architectes sont inscrits à l'Ordre des Architectes en 1996. Ces derniers se répartissent sur plusieurs modes d'exercice : 70,7\% exercent en libéral, 12,9\% en société, 8,4\% comme salariés d'entreprises et $3,3 \%$ comme fonctionnaires du secteur public. Ces chiffres sont toutefois sousestimés, compte tenu de la réticence croissante des architectes à s'inscrire à l'Ordre. L'INSEE quant à lui, qui dénombre les architectes non comme des individus mais comme des entreprises de services, arrive au chiffre de 24699 entreprises en 1995, dont une très large majorité (15 866) ne possédant pas de personnel salarié (architecte travaillant seul). 
carnet de commandes et de leur réputation, et comme s'ils étaient totalement déterminés par le contexte à être là où ils sont, indépendamment de leur propre action pour s'y placer.

Pour tenter de compléter cette compréhension statique des positions, nous rendrons compte d'une recherche qui s'est centrée sur le mode de constitution de ces positions (Biau, 1994). Dans ce travail, il s'agissait d'appréhender les pratiques de positionnement des architectes, c'est-à-dire leurs tentatives, leurs stratégies plus ou moins conscientes pour acquérir une position professionnelle plus confortable, pour approcher de leur modèle de réussite professionnelle : obtenir des commandes, se faire connaître ou, mieux, se faire reconnaître par les instances de légitimation à l'œuvre. On distinguera plusieurs familles de comportement, établissant des hiérarchies différentes quant aux enjeux, et on s'interrogera sur l'éventuelle mise en place, surtout chez les jeunes architectes, d'une trajectoire «canonique », faisant se succéder des étapes répertoriées, pour accéder aux sommets de la reconnaissance symbolique.

\section{IMAGES DES POSITIONS PROFESSIONNELLES CHEZ LES ARCHITECTES}

À plusieurs reprises dans les travaux sociologiques sur les architectes publiés au cours des vingt dernières années en France, des typologies ont été proposées pour rendre compte de la diversité des architectes tant dans leurs pratiques professionnelles que dans leurs systèmes de valeurs.

En 1973, R. Moulin, J.-P. Martinon et leurs collègues (Moulin et al., 1973) définissaient les positions héritées de l'académisme en distinguant quatre types-idéaux de praticiens. Ils différenciaient :

1) les mandarins traditionalistes, lauréats du Prix de Rome pour la plupart, détenteurs monopolistiques de la commande de monuments et tenants d'une conception de l'architecte-artiste seul à même de signer une création, chef d'orchestre veillant au bon respect des intentions esthétiques dans la réalisation de l'œuvre ;

2) les princes ou mandarins modernistes, proches du discours des précédents mais se tenant à l'écart des distinctions académiques et élargissant la mission légitime de l'architecte à l'intervention sur le logement et la ville ;

3) les architectes-patrons, ou architectes d'affaires, intégrant les conditions nouvelles de rationalisation et de division du travail autant que les exigences de rentabilité des promoteurs, et, enfin,

4) les utopistes et sorciers, se plaçant en marge d'un marché qu'ils désapprouvent et dont les productions principales sont l'image et le discours.

Mais au cours des vingt années qui nous séparent de l'époque de cette analyse, les évolutions ont été nombreuses et d'importance : le système académique qui existait encore alors s'est effacé, laissant plus de place à un débat idéologique et doctrinal sur l'architecture et sa place dans la société. Quant aux « architectes de papier », utopistes et sorciers, ils ont quasiment disparu, sauf à les redéfinir comme ceux dont l'essentiel de l'activité est actuellement de produire des images architecturales et urbanistiques non suivies de réalisations. Le volume global de la commande a, pendant ces deux décennies, subi diverses crises graves et les architectes-patrons, à 
la tête des très grandes agences françaises, ont dû réduire drastiquement leurs effectifs et élargir leur palette de commanditaires.

Aussi, en 1989, dans son Esquisse du champ de l'architecture fondée sur l'analyse de trajectoires professionnelles, l'équipe de J. Allégret (Allégret et al., 1989) propose une nouvelle typologie d'architectes s'appuyant d'une part sur les caractéristiques de leur production et d'autre part sur la nature des instances et institutions dont ils se recommandent. Cet ouvrage différencie ainsi

1) les architectes d'avant-garde, proches des avant-gardes artistiques, qui produisent une architecture avant tout destinée à leurs pairs et qui sont souvent membres des instances de sélection et de reconnaissance du champ de l'architecture ;

2) les producteurs «d'art bourgeois » qui sont attentifs à une demande sociale de patrimonialité et de classicisme ; souvent hautement qualifiés en archéologie et en techniques du bâtiment, ils sont proches des institutions publiques et parapubliques de conservation des monuments historiques et du bâti ancien ;

3) les producteurs de grande consommation, les plus nombreux, qui traitent la commande ordinaire et mettent au premier plan l'économie et le confort dans leurs choix de conception.

En se situant moins, comme R. Moulin ou comme J. Allégret, sur le terrain des pratiques (position par rapport à l'organisation du travail, à la commande, position par rapport aux instances, position esthétique et caractéristiques de leur production) que sur celui des représentations, des systèmes de références, des définitions de l'expertise professionnelle en architecture, I. Benjamin et F. Aballéa (Benjamin, Aballéa, 1990) ont quant à eux mis au jour deux « professionnalités » opposées. Il y aurait selon eux celle des «architectes-créateurs », dont la référence se trouve davantage du côté de l'art que de celui de la technique, même s'ils ont conscience de travailler dans un contexte particulier de création par rapport aux artistes plasticiens puisque l'on exige d'eux qu'ils satisfassent à des exigences fonctionnelles, économiques et constructives. Méfiants vis-à-vis de la commande privée, ils trouvent dans le système des concours les conditions optimales d'expression stylistique et d'innovation. À l'opposé, se trouverait le registre des «architectes-médiateurs », ceux qui adoptent volontiers le discours d'une architecture «modeste mais sympathique » et privilégient la satisfaction du client sur l'originalité de leur production. Ces derniers sont attentifs à l'émergence de nouvelles demandes de services et adaptent rapidement leurs modes de travail aux évolutions organisationnelles et techniques de leur secteur d'activité.

On le voit, quand l'on met en parallèle ces définitions des positions diverses voire antagonistes dans l'espace de la production architecturale, divers critères de lecture se combinent. L'analyse de R. Moulin donne beaucoup de poids à la relation de l'architecte avec les instances de pouvoir symbolique concernant la profession : affiliation à l'académisme ou à ce qu'il en reste à l'époque de sa recherche, position idéologique militante en opposition à celui-ci, ou encore désintérêt pour ce débat et spécialisation sur les aspects les plus pragmatiques (architectes d'affaires) ou au contraire les plus abstraits («sorciers») de la discipline. Du point de vue de J. Allégret, c'est le type de reconnaissance attendue qui différencie les praticiens : reconnaissance des milieux artistiques et intellectuels pour les «producteurs 
d'avant-garde », dépendance à l'égard des élites économiques pour les «producteurs d'art bourgeois» ou encore rapprochement avec les logiques technico-économiques et les milieux artisanaux du bâtiment pour les «producteurs de grande consommation ». L'opposition créateur/médiateur enfin, telle que la propose F. Aballéa, fait largement appel aux représentations du savoir-faire légitime de l'architecte et fait apparaître le clivage entre praticiens travaillant principalement pour la commande publique et praticiens vivant essentiellement de commandes privées.

\section{LES ENJEUX DU POSITIONNEMENT DANS LE CHAMP ACTUEL DE L'ARCHITECTURE}

Il est évident de dire que l'activité architecturale fait l'objet d'au moins deux grands enjeux: un enjeu économique (obtenir des commandes), un enjeu symbolique (être reconnu comme un architecte de qualité). Avant de se préoccuper de sa «cote» professionnelle, l'architecte s'inquiète bien sûr de trouver suffisamment d'affaires pour rétribuer son travail et celui des salariés qu'il emploie éventuellement. Quelques données statistiques donnent la mesure de la concurrence qui règne en architecture et donc de l'acuité de l'enjeu économique. L'Ordre des Architectes affiche pour 1995 un taux de chômage dans la profession de 20\%, ce qui signifie que le cinquième des praticiens inscrits et cotisant à cette instance n'ont eu aucune activité dans leur domaine au cours de l'année. On savait par ailleurs que le revenu moyen de l'architecte libéral se trouvait loin derrière celui des autres professions libérales (environ la moitié de celui des avocats et des dentistes). En effet, si les effectifs d'architectes ont plus que triplé au cours des vingt dernières années, passant d'environ 8000 en 1973 à 26500 en 1994, le nombre des logements mis en chan-tier ${ }^{3}$ pendant cette même période est quant à lui passé de 570000 à $302000^{4}$. On complétera ces quelques indications par la disparité de la répartition de la commande selon l'âge de l'architecte : $70 \%$ du chiffre d'affaires global de la profession sont réalisés par les architectes de 45 ans et plus (qui constituent un peu moins de la moitié des effectifs), alors que les architectes de moins de 45 ans se partagent les $31 \%$ restants ${ }^{5}$.

Par rapport à l'enjeu de la commande, il est assez délicat de situer l'enjeu symbolique, celui de se faire connaître/reconnaître. À première vue, l'un et l'autre semblent fonctionner dans une grande indépendance : il suffit par exemple d'observer que les architectes français dont le volume de commandes est actuellement le plus élevé sont quasiment absents du débat architectural comme des pages consacrées à l'architecture dans les supports d'information non spécialisés. L'architecture suit en cela l'opposition très connue dans les domaines artistiques entre le succès commer-

3. Le nombre de logements mis en chantier n'est qu'un indicateur partiel du marché des architectes puisqu'il ne représente qu'un segment de l'activité de construction et précisément celui dans lequel les architectes sont le moins présents. Mais il est l'une des seules données comparatives dont nous disposions pour donner une idée de l'évolution du volume de la construction en France au cours des vingt dernières années.

4. Pour 1973, nous reprenons les chiffres cités par Henry Salmon dans son rapport « Architecture et Cadre de vie », Journal Officiel n5, 2 avril 1988. Pour 1994, les chiffres sont issus du Bulletin de Conjoncture n²4, décembre 1995 émanant du Conseil National de l’Ordre des Architectes.

5. D'après les chiffres publiés en 1993 par le Conseil National de l'Ordre des Architectes. 
cial d'une œuvre et sa reconnaissance par la critique spécialisée. Un certain nombre des instances et des processus qui ont été identifiés, dans le champ de l'architecture, pour leur rôle dans la production de la valeur symbolique des œuvres et de leurs concepteurs, ont d'ailleurs de forts liens de parenté avec celles et ceux qui ont cours dans les champs artistiques : prix, expositions, publications, accès à des postes-clés dans l'enseignement, dans les jurys, les appareils administratifs ou les organes de la critique architecturale (revues, galeries d'exposition...), etc. Mais comme dans le domaine des arts plastiques, il n'y a pas une simple accumulation des diverses formes de rétribution de la qualité architecturale : l'importance quantitative d'une œuvre n'implique ni sa valorisation par la critique, ni sa notoriété dans le grand public.

La grande commande publique d'architecture semble toutefois être en situation d'articulation entre ces variables : elle associe à la rémunération matérielle du travail une valorisation symbolique de l'œuvre et de son créateur, dans le champ professionnel d'abord et dans le grand public éventuellement par la suite.

Il se trouve en effet qu'en France, pour des raisons historiques qu'il serait long d'exposer ici avec la finesse nécessaire, la commande d'édifices publics émanant de l'État, des collectivités locales et des organismes de logement social joue traditionnellement un rôle tout à fait particulier dans le champ de l'architecture. Jusque dans les années 1970, la commande publique reposait, comme l'avaient bien analysé R. Moulin, G. Lagneau et J. Lautman (Moulin et al., 1970), sur une oligarchie de corps et de listes d'architectes : au sommet du prestige se tenait le corps des Architectes des Bâtiments Civils et des Palais Nationaux, largement composé de lauréats du Grand Prix de Rome. Leur très faible nombre ne suffisant pas à répondre à la demande accrue de l'après-guerre pour la réalisation de logements et d'équipements publics, les administrations se sont aussi constitué des «viviers » d'architectes sous forme de listes d'architectes agréés, exerçant à titre libéral.

Au cours du bouleversement profond des légitimités qui s'est opéré dans les années suivant immédiatement 1968, ces positions acquises ont été remises en cause. Un système de concours s'est mis progressivement en place, destiné à ouvrir plus largement l'accès des praticiens à la commande publique et, en même temps, à améliorer la qualité et à promouvoir l'innovation dans ce domaine de la construction. Les dispositions légales se sont succédées pour fixer des procédures et des seuils. On ne retiendra que le principe actuellement en vigueur: pour une commande entraînant plus de 900000 F T.T.C. d'honoraires, le maître d'ouvrage doit organiser un concours et indemniser les candidats qu'il aura retenus pour concourir; si le montant des honoraires est compris entre 450000 et $900000 \mathrm{~F}$ T.T.C., le maître d'ouvrage doit publier un avis de candidature et désigner la commission multipartite qui sélectionnera un concepteur au vu des dossiers de références qui lui auront été fournis. En-deça de 450 000F T.T.C. d'honoraires, le maître d'ouvrage est libre de ses négociations.

Environ 800 concours ont été organisés en 1988 et 1200 en 1992 sur l'ensemble du territoire français (on n'englobe pas dans ces chiffres les simples sélections sur références correspondant au deuxième cas évoqué). Si l'on considère que, d'autre part, un concours suscite toujours au moins une cinquantaine de candidatures, dont 4 ou 5 retenues pour élaborer une esquisse ou un avant-projet, on prend toute la 
mesure de l'activité professionnelle et des enjeux qui sont liés aux concours de maîtrise d'œuvre publique.

Du diagnostic même de l'instance publique garante de l'équité et de l'efficacité de cette procédure d'attribution de la commande ${ }^{6}$, les concours perpétuent une forte inégalité des chances : sur les 261 concours supervisés par la Mission Interministérielle pour la Qualité des Constructions Publiques entre janvier 1985 et décembre $1990^{7}$, on observe une nette tendance à la concentration des commandes sur un nombre restreint d'architectes. Sur cette période de cinq ans, dans des programmes comme les bâtiments scolaires ou les hôpitaux, environ 50\% des architectes retenus l'avaient déjà été une fois au moins antérieurement pour un programme semblable. Globalement, $60 \%$ des concours étudiés retenaient des architectes qui avaient déjà été retenus 3 fois et plus dans des concours sur un même type de programme. On tend donc à revenir, dans le cadre du système des concours, à ce qu'ils étaient supposés éviter : la spécialisation d'un sous-groupe des architectes sur la commande publique, et même la spécialisation de quelques architectes sur certains types de constructions publiques. Parallèlement, depuis la réforme de l'attribution de cette commande, et par rapport aux années d'après-guerre, l'évaluation de l'architecte s'est de plus en plus concentrée sur l'amont du processus de construction, le «concept», l'esquisse ou le projet, et de moins en moins sur la qualité et la durabilité de sa réalisation. Ainsi, le seul fait d'être lauréat d'un grand concours, même si le projet n'est pas réalisé, ou pas selon le projet initial, devient en soi une marque de reconnaissance, entraînant publications, expositions, etc.

\section{POUR UNE APPROCHE DES POSITIONNEMENTS STRATEGIQUES DES ARCHITECTES}

En se centrant sur ces enjeux de la commande et de la notoriété et sur la question de leur interaction, il paraissait intéressant de considérer comment les praticiens se comportaient dans des systèmes de références en apparente opposition, de prendre la mesure de ce qu'ils choisissent et de ce qu'ils subissent en la matière. Cette question ne pouvait évidemment pas être abordée de front : on connaît la réticence des architectes à évoquer la dimension stratégique et concurrentielle de leur activité. On a donc dû contourner cette difficulté en faisant porter l'enquête sur les pratiques de communication, avec l'intuition que, dans cet aspect apparemment secondaire et anodin de la pratique professionnelle, se jouaient en fait chez les architectes à la fois une observation, une interprétation et une réaction personnelles aux processus valorisant différentiellement les prestations architecturales, au plan économique d'une part, au plan symbolique d'autre part.

Cette enquête, menée en 1993-94 dans le cadre d'une recherche financée par le Plan Construction et Architecture (Ministère de l'Équipement, du Logement, des Transports et de l'Espace), devait pour des raisons matérielles se limiter à un effectif d'une vingtaine de praticiens. Or, pour la pertinence de l'approche, il était primordial de prendre connaissance de l'éventail le plus large possible des pratiques en question, et nos hypothèses nous conduisaient à les rechercher en faisant varier

6. Mission Interministérielle pour la Qualité des Constructions Publiques.

7. Architectures Publiques $n^{\circ} 20$, mai 1993, «Concours : tous les chiffres depuis 4 ans ». 
au maximum les types d'architectes et d'agences étudiés. Nous avons donc croisé les critères qui permettaient de couvrir la gamme la plus complète de situations dans cet échantillon réduit :

— l'âge de l'architecte et l'ancienneté de l'agence,

- la localisation géographique (Paris, grandes villes, petites villes de province),

- la taille de l'agence, la nature et le volume de ses commandes, qui ne pouvaient guère être connus a priori avec précision ${ }^{8}$,

- la notoriété, sous ses formes les plus diverses (réussite à des concours, publications dans la presse spécialisée ou générale, réussite à des prix, position connue dans la critique ou l'enseignement, etc.).

Nous avons ensuite usé d'annuaires, de catalogues d'exposition, de listes de prix et d'informations orales pour identifier les personnes à interroger. Les refus ont été exceptionnels.

Sur la base de la consigne «Comment faites-vous pour faire connaître votre travail à l'extérieur ? », chacun des architectes a pu décrire ses pratiques de valorisation professionnelle au cours d'entretiens d'une durée d'une heure en moyenne. En outre, un rapide questionnaire oral permettait de réunir et comparer divers éléments de caractérisation des positions actuelles et des trajectoires : effectifs de l'agence, type de commande traitée, lieu et date du diplôme, premier emploi, fonctions exercées à l'extérieur de l'agence (enseignement, expertises, représentation institutionnelle...).

Les architectes, autant que les discours recueillis permettent de l'appréhender, adoptent une grande diversité de stratégies du «paraître » : certains sollicitent des rendez-vous auprès de grands constructeurs, certains organisent des visites de leurs réalisations, certains fréquentent les vernissages d'expositions, tentent d'y rencontrer les critiques d'architecture, font un travail constant d'envoi de documents sur leurs travaux aux revues d'architecture; d'autres encore s'inscrivent dans des clubs ou associations leur permettant de tisser des liens amicaux avec des décideurs politiques et économiques... Certaines agences d'architecture se sont même dotées d'agents de communication à temps partiel ou complet pour donner plus d'efficacité à cette activité, jugée importante pour l'obtention de commandes.

En un point, toutes les démarches individuelles concourent cependant : tous sont amenés à se doter d'une plaquette, d'un recueil de petite dimension, où sont rassemblées des informations sur les projets et réalisations de l'architecte, seul ou en équipe : plans, croquis, photos, éventuellement accompagnés de commentaires et de données chiffrées (surfaces, coûts,...). On y trouve parfois aussi une présentation de l'agence: personnel, moyens informatiques... La diffusion du concours comme méthode de choix du concepteur dans le domaine de la commande publique mais aussi, de plus en plus, dans celui de la promotion privée, a largement contribué au cours des dernières années à faire de cet outil le minimum nécessaire pour l'architecte. Celui-ci doit en effet, pour poser sa candidature à une consultation ou à un concours, pouvoir présenter rapidement un recueil de ses références, si possible

8. La MAF, Mutuelle des Architectes Français, qui couvre les risques professionnels, dispose de cette information mais ne la délivre que de façon anonyme et globale. 
organisées en fonction du client potentiel et du type de prestation pour lequel il concourt. C'est aussi le document qui peut servir d'appui aux visites que rendent certains architectes à des entreprises, des institutions auxquelles ils se présentent spontanément, espérant être consultés dès le prochain projet immobilier.

Plutôt que de décrire pour elles-mêmes ces diverses pratiques, il semblait intéressant d'analyser leur contenu symbolique pour les architectes. Les travaux classiques de l'anthropologie et du structuralisme ont depuis bien longtemps montré la prégnance des couples d'opposition dans les principes de génération des pratiques, principes valables aussi bien pour les pratiques quotidiennes que pour les rituels ou les mythes. Un certain nombre de couples d'opposition caractérisent en effet le discours par lesquels les architectes ont décrit et argumenté leurs comportements. Les deux principales oppositions peuvent être décrites, de manière simplifiée, de la manière suivante :

- Faire connaître sa production, en assurer la publication, faire du démarchage auprès de clients potentiels fait pleinement partie de la tâche de l'architecte et doit être mené avec professionnalisme, sérieux et continuité. Ou, au contraire, toute tâche autre que la conception et le suivi de la réalisation est une perte de temps pour l'architecte ; elle doit être réduite au strict nécessaire pour renouveler son carnet de commandes.

- Se faire reconnaître en tant qu'architecte, c'est obtenir la confiance de clients qui renouvelleront leur commande et propageront autour d'eux une appréciation positive du service que l'architecte a fourni. Ou, au contraire, se faire reconnaître en tant qu'architecte c'est avant tout se faire reconnaître par ses pairs, être crédité d'une personnalité dans sa façon de travailler, être présenté à la profession comme un créateur de référence, obtenir des publications, des distinctions, des prix.

Les prises de position individuelles des architectes pour l'une ou l'autre de ces opinions opposées deux à deux prennent tout leur sens quand on les confronte aux indicateurs objectifs de leur position par rapport à la commande et à la notoriété. Ainsi est-on amené à caractériser les comportements de positionnement en s'appuyant sur le schéma qui suit.

Ce schéma, réalisé à partir du petit échantillon sur lequel a porté la recherche, est construit pour mettre en relation les deux indicateurs qui nous intéressaient tout particulièrement : la notoriété, le volume de commandes. L'un comme l'autre ne peuvent s'appuyer sur des données fiables existantes. Pour ce qui concerne l'indicateur de la notoriété, nous avons retenu le nombre de publications dans les revues spécialisées, sans tenir compte de la presse générale qui, en France, n'ouvre que rarement ses colonnes aux architectes et qui, si elle le fait, se limite à un groupe très réduit d'architectes déjà consacrés par leur profession. L'importance des commandes traitées par les architectes n'est pas non plus aisément quantifiable : on a évoqué précédemment les disparités entre les commandes publiques et les commandes privées ; elles se doublent des différences entre les projets de bâtiments neufs et les projets de réaménagement de lieux existants et, bien sûr, de l'échelle de l'intervention et du budget qui s'y rapporte. On a donc essayé, au vu de la liste des œuvres des architectes analysés, de construire une échelle de 0 à 7 et de les y situer en tenant compte de tous ces critères. 


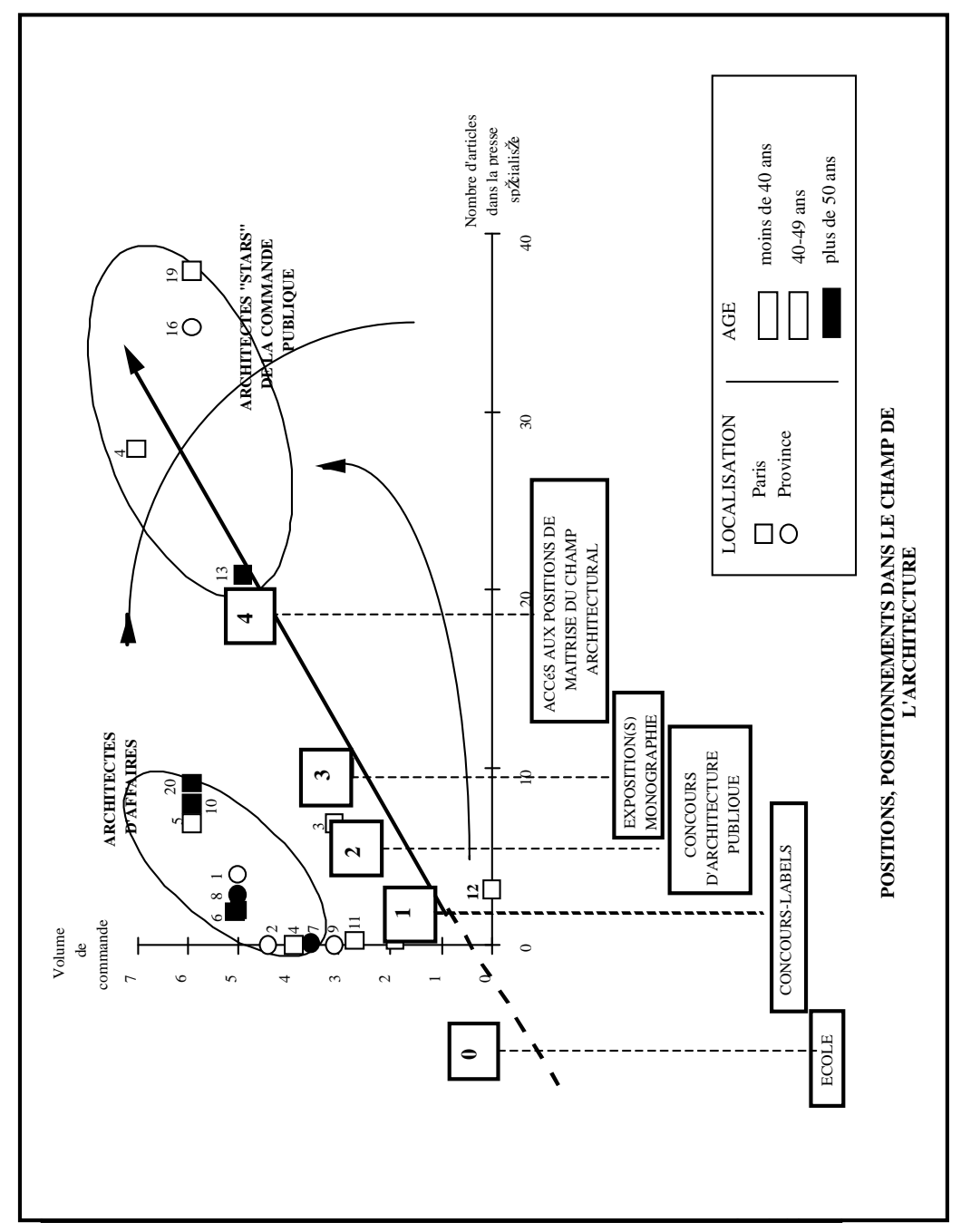


Dans les positions qui se dessinent, et que l'on vérifie actuellement sur un plus grand effectif, un clivage est déterminé par le nombre de publications sur les projets et réalisations : aucun architecte ne se trouve dans la fourchette comprise entre 10 et 20 publications. On peut voir là l'un des effets d'une presse architecturale qui, non dépourvue de souci commercial, tend à publier des architectes déjà connus et donc à renforcer les décalages entre un groupe réduit d'architectes médiatiques et la majorité des professionnels qui ne paraissent dans les revues que de manière accidentelle, voire jamais. Tout se passe comme s'il existait un seuil de notoriété en-deçà duquel, quel que soit l'avancement de sa carrière et l'importance de son œuvre, l'architecte ne suscite pas l'intérêt de la critique architecturale. Au-delà de ce seuil en revanche, les publications se multiplient rapidement, les grandes revues rivalisant souvent pour présenter la dernière réalisation de l'un des architectes en vue.

Pour ce qui est de la répartition sur l'échelle de la commande, on observe une gradation dans laquelle l'âge ne semble pas déterminant ${ }^{9}$. Ajoutons que, si l'on différencie commande publique et commande privée, on a, dans le groupe de droite, des architectes dont la commande publique représente entre 50 et $90 \%$ de leur activité, alors que le groupe de gauche présente à la fois des architectes parisiens construisant essentiellement pour le privé (les $n^{\circ} 5,6$ et 20) et des architectes ayant une activité assez également répartie sur ces deux secteurs mais qui sont établis en province.

Pour clarifier le propos, on a baptisé ces deux groupes selon des dénominations se référant à celles déjà avancées par nos prédécesseurs dans l'analyse des architectes : le groupe des architectes, très présents dans les revues professionnelles et développant une activité essentiellement tournée vers les constructions publiques a été nommé « les architectes-stars de la commande publique ». Le groupe des architectes traitant un volume de commandes notable mais dont la presse spécialisée ne fait qu'exceptionnellement écho a été intitulé «architectes d'affaires ». On notera que plusieurs individus se trouvent hors de cette dualité : des jeunes (les $n^{\circ} 3,9,12$, 15), dont la trajectoire professionnelle est encore incertaine, mais aussi des architectes plus âgés, dont la biographie professionnelle a connu des ruptures importantes (migration depuis l'étranger vers la France, depuis la province vers Paris, abandon récent du salariat pour l'exercice libéral par exemple).

À ce point, la question posée est la suivante : la diversité de ces positions trouvet-elle son homologue dans le registre des pratiques mises en œuvre par les architectes pour asseoir et améliorer leur position, vis-à-vis de leur accès à la commande d'une part, vis-à-vis de leur reconnaissance professionnelle de l'autre ?

Les architectes sont tous animés par le même enjeu, au-delà de l'obtention de commandes : celui de pouvoir choisir parmi différentes catégories de commandes. Mais parce que l'éthique professionnelle vise à minorer l'aspect mercantile de l'exercice libéral, cet enjeu qui se heurte, dans les représentations des professionnels, aux idéologies du non-profit et de l'architecture comme art n'est pas spontanément exprimé comme tel. On assiste alors chez les architectes à des

9. Cette remarque est bien sûr spécifique à l'échantillon étudié puisqu'au niveau national, comme on l'a dit plus haut, la répartition des chiffres d'affaires est très inégale selon les tranches d'âge des praticiens. 
compromis entre les idéaux professionnels et les réalités voire les nécessités du positionnement, compromis qui prennent deux formes :

- soit l'architecte déploie effectivement des pratiques de marketing quitte à les argumenter en d'autres termes,

- soit l'architecte se détourne des relations commerciales telles que les impose la commande privée. Il doit alors développer des stratégies différentes, mais bien réelles, pour accéder aux concours et, à travers eux, à la commande publique.

À la lecture de la presse professionnelle anglo-saxonne, on est frappé par la forte perméabilité des architectes aux formes de pensée et aux modalités de gestion issues du monde de l'entreprise. Des ouvrages ${ }^{10}$, des rubriques régulières, des articles diffusent des conseils de bon sens formulés comme des stratégies rationalisées, comportant diverses étapes répertoriées. Dans les milieux français de l'architecture en revanche, il est souvent de mise d'afficher une certaine indépendance voire du mépris pour les attentes et les critères d'appréciation de leurs prestations par leurs clients.

Parmi les praticiens de l'échantillon étudié, la démarche de type commercial est très développée, et prend des formes assez inventives, tant chez les «architectes d'affaires » que chez certains des jeunes et «prétendants ». En 1980 et après des débats houleux dans la profession, le Code des devoirs professionnels a levé l'interdiction traditionnelle à la publicité qui s'appliquait aux architectes au même titre qu'à tous les professionnels libéraux. Â part à la télévision, et à condition de les financer lui-même, l'architecte peut donc diffuser des messages publicitaires. Peu d'entre eux connaissent ce droit avec précision, encore moins l'utilisent, la plupart s'accordent à ne lui trouver aucune sorte d'efficacité sinon, peut-être, pour les maîtres d'œuvre qui s'adressent à la petite commande privée (maison individuelle, aménagement et réhabilitation de logements). En revanche, ils rivalisent de moyens pour nouer, avec les clients potentiels, des relations qui se placent sous un autre rapport que celui de donneur d'ordre à prestataire de service.

En architecture, tout individu peut être considéré comme un client potentiel et la démarche peut alors être, comme celle des interviewés $n^{\circ} 1$ et 2 , de prendre prétexte d'anniversaires, de fêtes de début d'année ou de travaux sur les locaux de l'agence pour organiser une journée portes ouvertes destinée aux voisins, confrères, entreprises de bâtiment partenaires et anciens clients. L'architecte $\mathrm{n}^{\circ} 5$, avec les moyens que lui donne une agence comptant 70 à 80 salariés, a pu porter cette idée à une échelle beaucoup plus importante : «On a fait un événement autour du lancement de cette maquette (d'un projet pour l'île Seguin à Boulogne). On a fait une croisière autour de l'île de Boulogne, il y avait des hommes politiques, des gens du monde de l'architecture, des promoteurs, des constructeurs, des industriels, des gens du cinéma, puisque l'île était dédiée au cinéma et aux technologies de

10. Deux livres, destinés à aider les architectes dans leur promotion, ont eu d'importants succès de librairie :

- celui de G. Jones, journaliste, anciennement chargé du marketing d'une grosse agence d'architecture et d'ingénierie aux USA : JONES (Gerre) How to market professional design services, Ed. Mac Graw Hill, 1983

- celui de J. Hook, qui a travaillé en agence et dans la presse architecturale en Grande-Bretagne : HOOK (James), Architects' marketing manual, People in, Harrogate, 1984. 
communication». Et il a, pour cette occasion, engagé une attachée de communication qu'il a ensuite conservée pour des tâches plus quotidiennes de relations de presse et de communication interne dans l'entreprise. La même agence, ainsi que celle de l'interviewé $n^{\circ} 2$, porte aussi une grande attention à ses panneaux de chantier, veille à ce que son nom y figure en gros caractères, et les complète éventuellement de banderoles et affichettes sur les vitrages.

Enfin, l'inscription au sein de réseaux sociaux choisis est, et reste surtout en province, une pratique courante. Aucun des interviewés n'a évoqué d'activité politique, mais en revanche, beaucoup appartiennent à des associations sportives ou culturelles, voire à des clubs (Rotary's ou Lyon's Club) dont les activités les amènent à fréquenter les chefs d'entreprise, les élus, les notables locaux. On sait aussi comment le fait plus ou moins hasardeux de se trouver à côté d'un député, d'un maire ou d'un grand décideur à l'occasion d'un voyage vers la capitale peut aider à entamer une collaboration.

On ne trouve rien sur ce thème dans les propos des « architectes de la commande publique », même concernant la partie de leur activité qui passe par des commandes privées. L'interviewé $n^{\circ} 19$ exprime clairement son attitude : «On considère que la seule démarche commerciale, c'est à la rigueur de faire savoir ce qu'on a fait, c'est tout. » Et il ajoute que la célébration étant beaucoup plus efficace quand elle est menée par une tierce personne, il préfère donner à la presse tous les moyens de l'obtenir d'elle plutôt que de l'assurer lui-même. L'architecte $n^{\circ} 4$ a une démarche similaire, quoiqu'il consacre un budget non négligeable en temps et en argent à accompagner ses clients dans des visites de réalisations à l'étranger. Son propos est de réunir les conditions d'une meilleure compréhension respective avec son client et de se donner des références communes : «On a eu un centre commercial très important à faire à l'agence. Eh bien, l'année dernière on est allés passer huit jours aux États-Unis, on a visité vingt-cinq centres commerciaux, avec les clients qui étaient là eux-mêmes et l'architecte aussi. C'est très utile parce qu'on jette un regard différent sur les choses, on peut échanger nos points de vue, nos critiques sur ce qu'on regarde, voire nos désaccords sur les jugements qu'on porte sur les bâtiments. En même temps, ce sont des occasions où vous avez accès à des personnes qui sont en général fort occupées à Paris. Là, vous les avez d'une manière plus détendue, donc on peut approfondir les discussions, on peut aller plus au fond des choses sur ce qu'on pense, c'est très utile les voyages. On l'a fait aux États-Unis, à Berlin, à Séville. On amenait souvent des gens en Angleterre.»

Cette démarche présentée comme «pédagogique» emprunte beaucoup aux moyens traditionnels du marketing mais la différence fondamentale est qu'elle prend place après la signature du contrat, et non dans la finalité de l'obtenir puisque, comme on l'a vu, l'accès à la commande publique suit un parcours plus complexe.

L'une des caractéristiques de la commande publique est en effet qu'elle permet aux architectes d'obtenir des commandes sans «se compromettre » dans des pratiques jugées trop mercantiles ${ }^{11}$. L'architecte $\mathrm{n}^{\circ} 12$, jeune, récemment installé à

11. On note d'ailleurs dans ces propos une inversion par rapport aux observations de l'équipe de R. Moulin en 1970 (op. cit.). Si aujourd'hui la commande publique est souvent présentée par les architectes comme une possibilité de travailler «en chambre » sans compromission, la commande publique d'il y a 25 ans était quant à elle synonyme de «relations », avec toute la connotation 
Paris après des études en province et des travaux temporaires chez d'autres architectes, n'a pas encore construit en son nom personnel. Mais il participe chaque année à trois ou quatre concours au moins et ne vit que des indemnités qu'il reçoit pour ces participations à des concours dont il n'a, à l'époque de notre entretien, jamais encore été lauréat. Il privilégie toutefois son indépendance par rapport au client : "Quand je fais un concours, j'envoie un book. Il y a un côté impersonnel à la chose. Les gens regardent les trois photos qu'il y a dessus, elles leur plaisent ou leur plaisent pas, mon nom leur dit ou pas quelque chose. Je fais mon projet dans mon coin, je rends ma copie et puis je suis jugé avec une discussion très réduite avec les maîtres d'ouvrage, on prend ou on ne prend pas. »

Compte tenu de l'abondance des candidatures, les architectes ont conscience que le système des concours n'est pourtant pas non plus totalement pur et dépourvu de compromissions : pour retenir l'attention parmi la cinquantaine de candidats et être admis à concourir contre trois ou quatre autres équipes, il est important d'être déjà connu d'un ou plusieurs membres du jury ${ }^{12}$. C'est ici que prend place la quête de reconnaissance symbolique auprès des confrères : plus que par des pressions personnelles auprès de tel ou tel participant à la décision, le contexte actuel amène l'architecte à s'imposer par sa réputation, sa « signature ». Or, en architecture comme dans des domaines de plus en plus nombreux de la vie sociale, une aura particulière est attribuée aux personnes et aux faits qui parviennent à entrer dans le système médiatique. Selon une association, voire une confusion, entre le fait d'être connu et celui d'être reconnu, les architectes faisant l'objet d'expositions, de publications, d'émissions télévisées se voient attribuer des qualités, une forme de reconnaissance symbolique, dont les critères d'évaluation ne sont jamais clairement énoncés mais dont les effets sont certains (Biau, 1992). Les pouvoirs publics responsables de la profession ne s'y sont pas trompés, multipliant les actions symboliques de promotion des jeunes architectes (Albums de la Jeune Architecture et Programmes d'Architecture Nouvelle mais aussi affiches, expositions, concours, prix consacrés aux jeunes architectes, etc.).

De ce fait, un enjeu majeur du travail relationnel et/ou médiatique déployé par l'architecte pour se faire connaître est de faire reconnaître sa compétence professionnelle par ses pairs. C'est dans cet esprit que les architectes entretiennent des relations suivies avec les rédacteurs des principales revues d'architecture, les invitant à visiter leur dernière réalisation, envoyant régulièrement des propositions d'articles sur leurs projets, ne négligeant pas les occasions de rencontre « fortuites » avec ces critiques (vernissages d'exposition, voyages d'études,...) ou mettant à profit les relations familiales ou amicales permettant de s'approcher de ce milieu, très réduit et très parisien. L'enseignement, la recherche, le fait de produire des

péjorative liée à l'apparence d'arbitraire dans l'ajustement entre offreurs et demandeurs. L'ouvrage cité montre bien en effet les liens entre d'une part des fonctionnaires divisés par corps, écoles, champs d'activités, etc., en position de passer des commandes et d'autre part des architectes euxmêmes divisés par des concurrences économiques, des titres, des appartenances à des écoles et patronages.

12. Un jury de concours rassemble généralement une quinzaine de membres dont, selon la loi, un tiers au moins de praticiens de la maîtrise d'œuvre (architectes, ingénieurs, spécialistes de domaines techniques). 
conférences destinées à un public de spécialistes jouent aussi un rôle important dans ce sens.

Dans le discours du groupe des « architectes-stars de la commande publique » revient fréquemment le fait qu'ils n'agissent dans ce domaine que sur sollicitation extérieure. C'est très explicitement le cas de l'interviewé $\mathrm{n}^{\circ} 13$ qui a un parcours professionnel très atypique puisqu'il s'est d'abord fait une renommée nationale voire internationale de critique, d'auteur d'essais et d'enseignant de l'architecture avant d'arriver assez tardivement à l'exercice libéral, notant d'ailleurs combien cette image «d'intellectuel» avait pu nuire à sa crédibilité comme maître d'œuvre. Par rapport au système des concours et des publications, dans lequel il intervient plus naturellement du côté des évaluateurs que des évalués, il affiche une distance certaine : «On a fait quelques articles mais je ne fais pas d'effort pour me faire publier. Je ne suis pas à la mode et ça ne me gêne pas parce que j'accepte mal que l'architecture soit purement un phénomène de mode. Ça représente du boulot d'être à la mode ! Il y a une manière de présenter, de prendre des photos, de faire faire les perspectives et les dessins d'une certaine manière par des gens qui eux-mêmes sont à la mode etc. Ça exige tout un battage, un travail, de l'argent dépensé que moi je ne trouve pas utile de mettre là-dedans. En marketing je suis très mauvais et puis j'ai une espèce de répulsion. »

Les interviewés $\mathrm{n}^{\circ} 4,16$ et 19 en revanche, tout en affirmant avoir franchi un seuil qui leur donne une certaine liberté vis-à-vis de ces nécessités, décrivent avec précision et fierté l'organisation de leur agence pour rationaliser leur accès aux concours et aux publications : conception de fiches par projet réalisé permettant de constituer rapidement un dossier de concours ou un dossier de presse, réalisation et duplication systématique de photographies de leurs constructions pour les publications, les expositions, les conférences, etc. Toutes ces tâches, faisant l'objet d'une très grande exigence, sont d'ailleurs souvent confiées à des spécialistes extérieurs.

Ce dispositif n'a pas son équivalent chez les « architectes d'affaires » qui, on l'a vu, n'ont d'ailleurs guère accès ni aux publications, ni aux concours. S'ils ont toutefois des stratégies dans ce sens, ce sont des stratégies de niveau préalable : ils ne sont pas encore dans le jeu et cherchent d'abord à se faire admettre parmi les joueurs. Ainsi l'architecte $n^{\circ} 5$ (49 ans) qui, après avoir dirigé l'une des sept plus importantes agences françaises, subit les effets de la crise de l'immobilier de bureaux, cherche à tout prix à diversifier son activité et à aborder la commande publique. Connaissant la faiblesse de ses chances s'il se présente d'emblée à un concours (compte tenu de la spécialisation des architectes de concours telle qu'elle a été évoquée plus haut), il applique aux maîtres d'ouvrage publics la stratégie de marketing qu'il connaît bien dans le privé : «On va établir un répertoire des administrations que l'on peut toucher, les noms des décisionnaires, le catalogue des personnes qui comptent dans les administrations, quelques noms et directions à travailler (sic). Ça va se faire par une enquête, du téléphone, du relationnel. »

De même, l'architecte $n^{\circ} 10$ (86 ans), qui ne trouve pas ses idées et ses goûts représentés dans les médias architecturaux actuels, a décidé de fonder avec des amis une nouvelle revue d'architecture de façon à se doter d'une tribune que les revues existantes ne lui accordaient pas : «On a voulu que cette revue ne soit pas spécialisée mais au contraire s'intéresse à toutes sortes de projets, toutes sortes de préoccu- 
pations, soit sur le plan esthétique, soit sur le plan technique, qu'il n'y ait pas qu'une seule tournure d'esprit comme on le voit maintenant. »

Quant aux architectes jeunes ou «prétendants » de notre échantillon, ils se montrent assez sensibles à l'enjeu de la reconnaissance par la critique architecturale et, selon leur expérience, s'organisent avec plus ou moins de succès pour s'y mesurer. La plus délibérée dans ce type de stratégie est l'architecte $\mathrm{n}^{\circ} 15$, jeune femme de 38 ans qui explique l'origine de sa réussite professionnelle par une campagne médiatique qu'elle a orchestrée avec le support de revues féminines. Elle explique dans l'entretien avoir alors joué avec les atouts dont elle disposait : sa réussite récente au concours des Albums de la Jeune Architecture, mais surtout sa féminité qui, associée au look quelque peu provocant qu'elle a adopté, en faisait un sujet idéal de portrait pour les magazines. Plus traditionnelle, et plus austère aussi, est la démarche de l'architecte d'origine allemande $\mathrm{n}^{\circ} 3$ qui, tirant l'enseignement de premières expériences difficiles dans la présentation orale de projets de concours au jury, ou de réalisations aux journalistes, s'est résolu à préparer scrupuleusement ces prestations, voire même à s'y faire assister par des partenaires extérieurs (philosophe, plasticien).

\section{VERS LA MISE EN PLACE D'UNE CARRIERE CANONIQUE EN ARCHITECTURE?}

Cette recherche amène à s'interroger sur un processus nouveau à propos duquel nous ne pouvons encore avancer que des hypothèses : cette reconnaissance symbolique semble se construire par étapes successives, fortement dépendantes chacune de celle qui la précède, en une forme de «carrière canonique » débouchant vers les plus hauts degrés de la consécration. Cette carrière est présente dans les représentations que se font les architectes de la réussite professionnelle, surtout chez les plus jeunes, qu'ils aient eux-mêmes déjà gravi certains de ses échelons ou non. Ainsi, les enjeux partent de la scolarité, au cours de laquelle il est important de suivre les enseignements d'architectes eux-mêmes bien placés dans la critique architecturale, souvent parisiens. Cette condition, si l'étudiant se révèle intéressant, peut faciliter l'accès à une deuxième étape : l'apprentissage précoce du savoir-faire lié aux concours, en général à l'occasion de « charrettes » ${ }^{13}$ dans l'agence privée du professeur ou de confrères. En parallèle, le jeune architecte au fait des instances dominantes de sa profession se porte souvent candidat aux «concours-labels » déjà évoqués. La réussite à ces concours ne conduit que rarement à une première réalisation mais à des publications ou des expositions qui peuvent susciter l'intérêt des spectateurs et qui, de plus, alimentent le press book qui est au jeune architecte ce que le curriculum vitae est au salarié. Ensuite la progression symbolique de l'architecte ayant franchi ces premières embûches repose sur deux phénomènes qui s'alimentent réciproquement.

13. Ce terme, en passe d'entrer dans la langue courante, désigne les périodes de travail intense qui précèdent une date impérative de rendu du travail, souvent un projet de concours. Il provient du jargon de l'École des Beaux-Arts, au moment où les étudiants, disséminés dans de multiples ateliers du Quartier Latin, devaient déposer leur travail dans la charrette qui parcourait les rues de ce quartier pour les collecter au soir de la date limite, faute de quoi ils ne pouvaient pas être pris en compte. 
- les concours : la caution que représentent les expériences dans des agences prestigieuses et la réussite à des concours patronnés par le Ministère de l'Équipement facilitent l'accès des jeunes architectes aux concours de maîtrise d'œuvre. Si, la première fois, le maître d'ouvrage peut retenir un architecte non confirmé pour son originalité et le talent montré dans la conception, faisant preuve par là d'ouverture d'esprit et de bonne volonté pour la promotion de la jeune architecture, il lui confiera généralement un programme assez restreint.

- la critique architecturale : toujours très attentive aux constructions publiques ${ }^{14}$ et parfois sollicitée par l'architecte, elle donnera plus ou moins de résonance à ses réalisations (publications dans les revues, expositions,...). Cette forme de publicité joue ensuite à l'appui des candidats et l'architecte dont le nom est connu est plus aisément retenu pour un concours important.

$\mathrm{Au}$ terme de cette spirale généralement ascendante, l'architecte se trouve non plus en position de solliciter publications, commandes et postes honorifiques mais de choisir entre ceux-ci. L'architecte Jean-Paul Viguier $\left(\mathrm{n}^{\circ} 4\right)$, dont l'agence était dans les années 1992-1993 l'une des plus importantes de France et qui a été choisi pour réaliser le pavillon de la France à l'Exposition Universelle de Séville en 1992, l'exprime sans détours : «Dans vos rapports avec les maîtres d'ouvrage, vous sentez qu'il y a un moment où ce que vous dites, ce que vous faites, est regardé avec plus d'intérêt qu'avant (...). Vous sentez bien que ce basculement psychologique du maître d'ouvrage face à vous est lié à tout l'environnement. L'aura, que vous avez par ailleurs, il ressent ça le maître d'ouvrage : on a parlé de vous là, il a vu une exposition, il a lu un livre où vous avez eu un de vos projets, il vient de voir dans le dernier magazine un projet de vous... Tout ça contribue à créer autour de vous une aura à laquelle les maîtres d'ouvrage sont très sensibles. »

C'est alors que devenant juré de concours, enseignant, membre de comités de rédaction etc., il reproduit sur les praticiens ascendants les critères d'évaluation qui l'ont fait lui-même parvenir à la consécration. Le système se boucle ainsi, reproduisant d'assez près le système académique traditionnel renversé en 1968.

On le voit, l'approche des stratégies de positionnement des architectes par leurs pratiques de présentation de soi ne remet pas en cause la dualité traditionnellement posée entre architectes-hommes d'affaires et architectes-artistes. L'éthique professionnelle de l'architecte continue en effet à se définir largement en fonction de l'opposition entre l'architecture comme production et l'architecture comme œuvre. Et largement aussi, ses efforts d'auto-promotion le tendront vers l'un ou l'autre pôle d'enjeux. Toutefois, elle précise les contours de l'action individuelle dans un contexte où la hiérarchisation symbolique des architectes semble bien emprunter des voies de plus en plus univoques et organisées, celles de la carrière canonique que l'on a évoquée plus haut.

Deux registres de questionnement ressortent de cette analyse. Le premier vient de l'observation que, tant dans la définition que dans le contrôle du déroulement de ces carrières d'élite, l'État joue un rôle central. C'est l'État qui assume la tutelle de

14. Les revues d'architecture consacrent une très large prédominance aux projets et réalisations financés par la maîtrise d'ouvrage publique. À titre d'exemple, l'Année du Moniteur, numéro spécial annuel de la grande revue d'architecture Le Moniteur Architecture-AMC, a consacré, depuis sa première livraison en 1989 , plus de $95 \%$ de ses articles à des constructions publiques. 
l'enseignement de l'architecture, c'est lui aussi qui organise les concours-labels qui jouent d'une certaine façon le rôle de «super-diplômes ». C'est aussi la sphère des administrations, des entreprises publiques et des collectivités locales qui contrôle le système des concours à partir duquel s'attribue une commande qui représente le quart de l'activité du bâtiment et près de la moitié des honoraires globaux de la profession. Mais c'est aussi l'État et les agents publics qui, clients éclairés, donnent aux architectes les conditions de produire les œuvres sur lesquelles la critique architecturale porte la plus vive attention. Il existait en France une longue tradition d' « architecture d'État», que la Reconstruction puis le développement urbain des années 1950-1970 avait entretenue. La décentralisation administrative au profit des maires a pris le relais au cours des années 1980-90. Mais on peut se demander, paradoxe d'une profession qui se range si spontanément sous l'étiquette de «profession libérale », si les instances publiques ne sont pas en passe de se substituer aux instances de la profession pour désigner les élites professionnelles.

Un deuxième questionnement s'attache à la dualité des stratégies que l'on a montrée ici, et, à travers elles, à la dualité des modèles de réussite professionnelle qui animent les praticiens. Si l'on comprend la différenciation des stratégies, il nous reste des interrogations sur leur aboutissement. En empruntant le vocabulaire de P. Bourdieu, on peut dire que l'on observe d'un côté des stratégies d'accumulation d'un capital économique et de l'autre, des stratégies d'accumulation d'un capital symbolique qui, bien sûr, se monnaie lui aussi. On est tenté de dire, d'un point de vue peut-être partial, que cette deuxième forme de capital est plus enviable que la première, dont elle n'est d'ailleurs pas exclusive. Cette opinion se renforce de quelques propos tenus par certains des architectes d'affaires interviewés qui, par divers arguments, ont signifié leur désir de s'approprier quelques-uns des bénéfices des architectes-stars : réaliser une grande opération publique (architecte $n^{\circ} 5$ ), être cité comme auteur de ses réalisations, au moins à parité avec le maître d'ouvrage (architecte $n^{\circ} 2$ ), avoir enfin un ouvrage traitant de soi-même et de ses réalisations (architecte $n^{\circ} 20$ ). Il semble en tout cas qu'il existe, pour des architectes très bien placés dans la sphère privée de l'immobilier et auteurs d'une production abondante, une extrême difficulté à se rapprocher, même en fin de carrière, des instances du pouvoir symbolique ; celles par exemple qui ont le pouvoir de définir l'architecture légitime et de faire émerger les architectes «de qualité », dignes d'être couronnés et de figurer dans les histoires et encyclopédies de l'architecture. Et peut-être n'y a-t-il pas de réel accomplissement professionnel pour l'architecte si la pérennité de son œuvre n'est que celle des édifices qu'il a réalisés au cours de sa carrière, et qu'il lui faille renoncer à faire entrer son nom dans le panthéon des grands architectes de la fin de ce siècle?

Véronique BIAU

Chercheur à l'École d'Architecture de Paris-La Défense LOUEST (UMR 220 du CNRS)

41, allée le Corbusier 92023 NANTERRE Cedex 


\section{RÉFÉRENCES BIBLIOGRAPHIQUES}

Allegret, J., Bertrand, R., DeBARRE, A., ACCORSI, F. 1989. Trajectoires professionnelles: esquisse du champ de l'architecture. Paris : École d'Architecture Paris-Villemin, 168 p.

Benjamin, I., ABAlleA, F. 1990. Évolution de la professionnalité des architectes. Recherche sociale $\mathrm{n}^{\circ} 113$, janvier-février 1990 , p. 3-56 et $\mathrm{n}^{\circ} 114$, avril-juin 1990, p. 57-109.

BERTRAND, R. 1988. Jacques Tournant : essai de trajectoire, contributif à une étude des trajectoires professionnelles des architectes français. Paris: École d'Architecture Paris-Villemin - BRA. 180 p.

BIAU, V. 1992. L'architecture comme emblème municipal ; les Grands Projets des maires. Paris : Plan Construction et Architecture.

BIAU, V. 1994. Comment se faire connaître et accéder à la commande; stratégies du paraître chez les architectes. Paris : Plan Construction et Architecture, 164 p.

BLAU, J. 1984. Architects and Firms ; a Sociological Perspective on Architectural Practice. Cambridge (Mass.), Londres : MIT Press. 189 p.

Boltanski, L., TheVenot, L. 1991. De la justification : les économies de la grandeur. Paris : Gallimard.

BOURDIEU, P. 1992. Les règles de l'art; genèse et structure du champ littéraire. Paris : Seuil.

GolzeN, G. 1984. How Architects get Work? (Interviews with Architects, Clients and Intermediaries). Londres: Architecture and Building Practice Design Guides.

HAUMONT, B. 1986. Figures salariales et socialisation de l'architecture. Paris : Ecole d'Architecture Paris-Villemin, coll. In Extenso, n ${ }^{\circ} .160$ p.

JACQUES, A. 1986. La carrière de l'architecte au XIX siècle. Paris : Éditions de la Réunion des Musées Nationaux. 72 p.

MARTINON, J.-P. 1974. Fonctions symboliques et types d'architectes. La Nouvelle Critique $\mathrm{n}^{\circ}$ spécial 78 bis.

MONTLIBERT de, C. 1995. L'impossible autonomie de l'architecte. Strasbourg: Presses Universitaires de Strasbourg, 1995.

Moulin, R. et al. 1973. Les architectes. Métamorphose d'une profession libérale. Paris : Calmann-Lévy. 311 p.

Moulin, R., Lagneau, G., Lautman, J. 1970. L'État et les architectes; essai d'analyse sociologique des systèmes de choix. Paris : Centre d'Ethnologie Française. 